УДК 512.535

\title{
S. BARDYLA
}

\section{AN ALTERNATIVE LOOK AT THE STRUCTURE OF GRAPH INVERSE SEMIGROUPS ${ }^{1}$}

S. Bardyla. An alternative look at the structure of graph inverse semigroups, Mat. Stud. 51 (2019), 3-11.

For any graph inverse semigroup $G(E)$ we describe subsemigroups $D^{0}=D \cup\{0\}$ and $J^{0}=J \cup\{0\}$ of $G(E)$ where $D$ and $J$ are arbitrary $\mathcal{D}$-class and $\mathcal{J}$-class of $G(E)$, respectively. In particular, we prove that for each $\mathcal{D}$-class $D$ of a graph inverse semigroup over an acyclic graph the semigroup $D^{0}$ is isomorphic to a semigroup of matrix units. Also we show that for any elements $a, b$ of a graph inverse semigroup $G(E), J_{a} \cdot J_{b} \cup J_{b} \cdot J_{a} \subset J_{b}^{0}$ if there exists a path $w$ such that $s(w) \in J_{a}$ and $r(w) \in J_{b}$.

1. Preliminaries. We shall follow the terminology of [11] and [19]. By $\mathbb{N}$ we denote the set of positive integers. The cardinality of a set $X$ is denoted by $|X|$. A semigroup $S$ is called an inverse semigroup if for each element $a \in S$ there exists a unique element $a^{-1} \in S$ such that $a a^{-1} a=a$ and $a^{-1} a a^{-1}=a^{-1}$.

By $\mathcal{R}, \mathcal{L}, \mathcal{J}, \mathcal{D}$ and $\mathcal{H}$ we denote Green's relations on a semigroup $S$ which are defined as follows: for each $a, b \in S$

$$
\begin{array}{lll}
a \mathcal{R} b & \text { if and only if } & a S \cup\{a\}=b S \cup\{b\} ; \\
a \mathcal{L} b & \text { if and only if } & S a \cup\{a\}=S b \cup\{b\} ; \\
a \mathcal{J} b & \text { if and only if } & S a S \cup a S \cup S a \cup\{a\}=S b S \cup b S \cup S b \cup\{b\} ; \\
& \mathcal{D}=\mathcal{L} \circ \mathcal{R}=\mathcal{R} \circ \mathcal{L} ; & \mathcal{H}=\mathcal{L} \cap \mathcal{R} .
\end{array}
$$

Let $S$ be a semigroup with zero $0_{S}$ and $X$ be a non-empty set. By $\mathcal{B}_{X}(S)$ we denote the set $X \times S \times X \sqcup\{0\}$ endowed with the following semigroup operation:

$$
(a, s, b) \cdot(c, t, d)= \begin{cases}(a, s \cdot t, d), & \text { if } b=c \\ 0, & \text { if } b \neq c,\end{cases}
$$

and $(a, s, b) \cdot 0=0 \cdot(a, s, b)=0 \cdot 0=0$, for each $a, b, c, d \in X$ and $s, t \in S$.

The semigroup $\mathcal{B}_{X}(S)$ is called the Brandt $X$-extension of the semigroup $S$. Obviously, the set $J=\left\{\left(a, 0_{S}, b\right) \mid a, b \in X\right\} \cup\{0\}$ is a two-sided ideal of the semigroup $\mathcal{B}_{X}(S)$. The Rees factor semigroup $\mathcal{B}_{X}(S) / J$ is called the Brandt $X^{0}$-extension of the semigroup $S$ and is denoted by $\mathcal{B}_{X}^{0}(S)$. If $S$ is the semilattice $(\{0,1\}$, min) then we denote the semigroup

The work of the author is supported by the Austrian Science Fund FWF (Grant I 3709 N35).

2010 Mathematics Subject Classification: 20M18.

Keywords: polycyclic monoid; graph inverse semigroup; Brandt $\lambda^{0}$-extension.

doi:10.15330/ms.51.1.3-11

(C) S. Bardyla, 2019 
$\mathcal{B}_{X}^{0}(S)$ by $\mathcal{B}_{X}^{0}$. The semigroup $\mathcal{B}_{X}^{0}$ is well-known (see page 86 from [19]) and is called the semigroup of $X \times X$-matrix units. Observe that semigroups $\mathcal{B}_{X}^{0}(S)$ and $\mathcal{B}_{Y}^{0}(S)$ are isomorphic iff $|X|=|Y|$. A Brandt $X^{0}$-extension of a group play an important role in the structure of primitive inverse semigroups (see [19, Chapter 3.3]). Algebraic and topological properties of a Brandt $X^{0}$-extension of a semigroup were investigated in [14] and [15].

For a cardinal $\lambda$ polycyclic monoid $\mathcal{P}_{\lambda}$ is the semigroup with identity 1 and zero 0 given by the presentation

$$
\left.\mathcal{P}_{\lambda}=\left\langle 0,1,\left\{p_{i}\right\}_{i \in \lambda},\left\{p_{i}^{-1}\right\}_{i \in \lambda}\right| p_{i}^{-1} p_{i}=1, p_{j}^{-1} p_{i}=0 \text { for } i \neq j\right\rangle .
$$

Observe that polycyclic monoid $\mathcal{P}_{0}$ is isomorphic to the semilattice $(\{0,1\}$, min). Polycyclic monoid is a generalization of the well-known bicyclic monoid (see [19, Chapter 3.4]). More precisely, the bicyclic monoid with adjoined zero is isomorphic to the polycyclic monoid $\mathcal{P}_{1}$. Polycyclic monoid $\mathcal{P}_{k}$ over a finite non-zero cardinal $k$ was introduced in [24]. Algebraic and topological properties of polycyclic monoids were investigated in $[4,9,10,13$, $20,21]$.

A directed graph $E=\left(E^{0}, E^{1}, r, s\right)$ consists of disjoint sets $E^{0}, E^{1}$ of vertices and edges, respectively, together with functions $s, r: E^{1} \rightarrow E^{0}$ which are called source and range, respectively. In this paper we refer to a directed graph simply as "graph". We consider each vertex as a path of length zero. A path of non-zero length $x=e_{1} \ldots e_{n}$ in a graph $E$ is a finite sequence of edges $e_{1}, \ldots, e_{n}$ such that $r\left(e_{i}\right)=s\left(e_{i+1}\right)$ for each positive integer $i<n$. By $\operatorname{Path}^{+}(E)$ we denote the set of all paths of a graph $E$ which have a non-zero length. We extend functions $s$ and $r$ on the set $\operatorname{Path}(E)=E^{0} \cup \operatorname{Path}^{+}(E)$ of all paths in the graph $E$ as follows: for each vertex $e \in E^{0}$ put $s(e)=r(e)=e$ and for each path of non-zero length $x=e_{1} \ldots e_{n} \in \operatorname{Path}^{+}(E)$ put $s(x)=s\left(e_{1}\right)$ and $r(x)=r\left(e_{n}\right)$. By $|x|$ we denote the length of a path $x$. Let $a=e_{1} \ldots e_{n}$ and $b=f_{1} \ldots f_{m}$ be two paths such that $|a|>0,|b|>0$ and $r(a)=s(b)$. Then by $a b$ we denote the path $e_{1} \ldots e_{n} f_{1} \ldots f_{m}$. If $a$ is a vertex and $b$ is a path such that $s(b)=a(r(b)=a$, resp.) then put $a b=b$ (ba=b, resp.). A path $x$ is called a prefix of a path $y$ if there exists a path $z$ such that $y=x z$. An edge $e$ is called a loop if $s(e)=r(e)$. A path $x$ is called a cycle if $s(x)=r(x)$ and $|x|>0$. Vertices $a$ and $b$ of a graph $E$ are called strongly connected if there exist paths $u, v \in \operatorname{Path}(E)$ such that $a=s(u)=r(v)$ and $b=s(v)=r(u)$. Define a relation $R$ on the set $E^{0}$ as follows: $(a, b) \in R$ if and only if vertices $a$ and $b$ are strongly connected. Simple verifications show that $R$ is an equivalence relation. Equivalence classes of the relation $R$ are called strongly connected components of a graph $E$. A graph $E$ is called acyclic if it contains no cycles.

For a given directed graph $E=\left(E^{0}, E^{1}, r, s\right)$ a graph inverse semigroup (or simply GIS) $G(E)$ over the graph $E$ is the semigroup with zero generated by the sets $E^{0}, E^{1}$ together with the set $E^{-1}=\left\{e^{-1} \mid e \in E^{1}\right\}$ which is disjoint with $E^{0} \cup E^{1}$ satisfying the following relations for all $a, b \in E^{0}$ and $e, f \in E^{1}$ :

(1) $a \cdot b=a$ if $a=b$ and $a \cdot b=0$ if $a \neq b$;

(2) $s(e) \cdot e=e \cdot r(e)=e$;

(3) $e^{-1} \cdot s(e)=r(e) \cdot e^{-1}=e^{-1}$;

(4) $e^{-1} \cdot f=r(e)$ if $e=f$ and $e^{-1} \cdot f=0$ if $e \neq f$.

Graph inverse semigroups are generalizations of the polycyclic monoids. In particular, for each cardinal $\lambda$ a polycyclic monoid $\mathcal{P}_{\lambda}$ is isomorphic to the graph inverse semigroup over the 
graph $E$ which consists of one vertex and $\lambda$ distinct loops. However, by [5, Theorem 1], each graph inverse semigroup $G(E)$ is isomorphic to a subsemigroup of the polycyclic monoid $\mathcal{P}_{|G(E)|}$.

According to [16, Chapter 3.1], each non-zero element of a graph inverse semigroup $G(E)$ can be uniquely represented as $u v^{-1}$ where $u, v \in \operatorname{Path}(E)$ and $r(u)=r(v)$. A semigroup operation in $G(E)$ is defined by the following way:

$$
\begin{aligned}
& u_{1} v_{1}^{-1} \cdot u_{2} v_{2}^{-1}=\left\{\begin{array}{cc}
u_{1} w v_{2}^{-1}, & \text { if } u_{2}=v_{1} w \text { for some } w \in \operatorname{Path}(E) ; \\
u_{1}\left(v_{2} w\right)^{-1}, & \text { if } v_{1}=u_{2} w \text { for some } w \in \operatorname{Path}(E) ; \\
0, & \text { otherwise, }
\end{array}\right. \\
& \text { and } u v^{-1} \cdot 0=0 \cdot u v^{-1}=0 \cdot 0=0
\end{aligned}
$$

Further, when we write an element of $G(E)$ in a form $u v^{-1}$ we always mean that $u, v \in$ $\operatorname{Path}(E)$ and $r(u)=r(v)$. Simple verifications show that $G(E)$ is an inverse semigroup and $\left(u v^{-1}\right)^{-1}=v u^{-1}$, for each element $u v^{-1} \in G(E)$.

Graph inverse semigroups play an important role in the study of rings and $C^{*}$-algebras (see $[1,3,12,18,25])$. Algebraic theory of graph inverse semigroups is well developed (see [2, $5,16,17,20,22])$. Topological properties of graph inverse semigroups were investigated in [6$8,23]$.

This paper is inspired by the paper of Mesyan and Mitchell [22] and can be regarded as an alternative look at the structure of graph inverse semigroups.

2. A local structure of graph inverse semigroups. By [22, Corollary 2], two non-zero elements $a b^{-1}$ and $c d^{-1}$ of a GIS $G(E)$ are $\mathcal{D}$-equivalent iff $r(a)=r(b)=r(c)=r(d)$. Observe that each non-zero $\mathcal{D}$-class contains exactly one vertex of $E$. By $D_{e}$ we denote the $\mathcal{D}$-class which contains vertex $e \in E^{0}$. Put $D_{e}^{0}=D_{e} \cup\{0\}$.

Lemma 1. Let $G(E)$ be a GIS, $a b^{-1} \in D_{e}^{0}$ and $c d^{-1} \in D_{f}^{0}$. Then $a b^{-1} \cdot c d^{-1} \in D_{e}^{0} \cup D_{f}^{0}$.

Proof. Fix any elements $a b^{-1} \in D_{e}^{0}$ and $c d^{-1} \in D_{f}^{0}$. If $a b^{-1} \cdot c d^{-1}=0$ then $a b^{-1} \cdot c d^{-1} \in$ $D_{e}^{0} \cup D_{f}^{0}$. Assume that $a b^{-1} \cdot c d^{-1} \neq 0$. Then there exists a path $w \in \operatorname{Path}(E)$ such that either $a b^{-1} \cdot c d^{-1}=a w d^{-1}$ or $a b^{-1} \cdot c d^{-1}=a(d w)^{-1}$. In the first case $c=b w$ which yields that $r(a w)=r(w)=r(c)=r(d)=f$. Hence $a b^{-1} \cdot c d^{-1} \in D_{f}$. In the second case $b=c w$ which implies that $r(d w)=r(w)=r(b)=r(a)=e$. Hence $a b^{-1} \cdot c d^{-1} \in D_{e}$.

Observe that if $u v^{-1} \in D_{e}$ then $\left(u v^{-1}\right)^{-1}=v u^{-1} \in D_{e}$ which provides the following:

Corollary 1. For each vertex e of a graph $E, D_{e}^{0}$ is an inverse subsemigroup of $G(E)$.

Further we need the following denotations. For any vertex $e$ of a graph $E$ put:

$$
\begin{gathered}
I_{e}=\{u \in \operatorname{Path}(E) \mid r(u)=e\} ; \\
Q_{e}=\left\{u \in I_{e} \mid r(v) \neq e, \text { for each non-trivial prefix } v \text { of } u\right\} ; \\
C_{e}=\left\{u \in I_{e} \mid s(u)=r(u)=e\right\} ; \\
C_{e}^{1}=C_{e} \cap Q_{e}=\left\{u \in C_{e} \mid r(v) \neq e, \text { for each non-trivial prefix } v \text { of } u\right\} .
\end{gathered}
$$

By $\left\langle C_{e}\right\rangle\left(\left\langle C_{e}^{1}\right\rangle\right.$, resp. $)$ we denote the inverse subsemigroup of $G(E)$ which is generated by the set $C_{e} \cup\{0\}\left(C_{e}^{1} \cup\{0\}\right.$, resp.). Observe that $e \in C_{e}^{1}$ and $e$ is the identity of the semigroup $\left\langle C_{e}\right\rangle$. The following theorem describes the structure of the semigroup $\left\langle C_{e}\right\rangle$. 
Theorem 1. For each vertex $e$ of any graph $E$ the semigroup $\left\langle C_{e}\right\rangle$ is isomorphic to the polycyclic monoid $\mathcal{P}_{\left|C_{e}^{1} \backslash\{e\}\right|}$.

Proof. Fix any vertex $e \in E^{0}$. Put $\lambda=\left|C_{e}^{1} \backslash\{e\}\right|$. Let $C_{e}^{1} \backslash\{e\}=\left\{u_{\alpha}\right\}_{\alpha \in \lambda}$. For convenience we denote $e$ by $u_{-1}$. It is easy to check that $\left\langle C_{e}^{1}\right\rangle=\left\{u v^{-1} \mid u, v \in C_{e}\right\} \cup\{0\}=\left\langle C_{e}\right\rangle$.

Let $G=\left\{p_{\alpha}\right\}_{\alpha \in \lambda} \cup\left\{p_{\alpha}^{-1}\right\}_{\alpha \in \lambda}$ be the set of generators of the polycyclic monoid $\mathcal{P}_{\lambda}$. Define a map $f:\left\langle C_{e}\right\rangle \rightarrow \mathcal{P}_{\lambda}$ consecutively extending it as follows. At first we define $f$ on $C_{e}^{1}$ by putting $f\left(u_{-1}\right)=1$ and $f\left(u_{\alpha}\right)=p_{\alpha}$ for each $\alpha \in \lambda$. Let $u \in C_{e} \backslash\{e\}$ be any element. It is easy to check that $u$ has a unique representation $u=u_{\alpha_{1}} u_{\alpha_{2}} \ldots u_{\alpha_{n}}$ as a product of elements of $C_{e}^{1} \backslash\{e\}$. We put $f(u)=p_{\alpha_{1}} p_{\alpha_{2}} \ldots p_{\alpha_{n}}$. Observe that any non-zero element of $\left\langle C_{e}\right\rangle$ has a unique representation in a form $u v^{-1}$ for some paths $u, v \in C_{e}$. Finally, put $f\left(u v^{-1}\right)=f(u) f(v)^{-1}$ and $f(0)=0$.

Obviously, $f$ is a bijection. Let us show that $f$ is a homomorphism. Fix any elements $a b^{-1}, c d^{-1} \in\left\langle C_{e}\right\rangle$. Let

$$
a=u_{\alpha_{1}} \ldots u_{\alpha_{n}}, \quad b=u_{\beta_{1}} \ldots u_{\beta_{m}}, \quad c=u_{\gamma_{1}}, \ldots u_{\gamma_{k}}, \quad d=u_{\delta_{1}} \ldots u_{\delta_{t}}
$$

be (unique) representations of elements $a, b, c, d$ as a product of elements of $C_{e}^{1}$ (here we agree that if some of the elements $a, b, c$ or $d$ are equal to $e$, then their representations are equal to $\left.u_{-1}\right)$. There are three cases to consider:

(1) $b$ is a prefix of $c$;

(2) $c$ is a prefix of $b$;

(3) $a b^{-1} \cdot c d^{-1}=0$.

Suppose that case 1 holds, i.e., $u_{\gamma_{1}} \ldots u_{\gamma_{k}}=u_{\beta_{1}} \ldots u_{\beta_{m}} u_{\gamma_{m+1}} \ldots u_{\gamma_{k}}$. Observe that

$$
a b^{-1} \cdot c d^{-1}=u_{\alpha_{1}} \ldots u_{\alpha_{n}} u_{\gamma_{m+1}} \ldots u_{\gamma_{k}}\left(u_{\delta_{1}} \ldots u_{\delta_{t}}\right)^{-1} \text {. }
$$

Then

$$
\begin{gathered}
f\left(a b^{-1} \cdot c d^{-1}\right)=f\left(u_{\alpha_{1}} \ldots u_{\alpha_{n}} u_{\gamma_{m+1}} \ldots u_{\gamma_{k}}\left(u_{\delta_{1}} \ldots u_{\delta_{t}}\right)^{-1}\right)= \\
=p_{\alpha_{1}} \ldots p_{\alpha_{n}} p_{\gamma_{m+1}} \ldots p_{\gamma_{k}}\left(p_{\delta_{1}} \ldots p_{\delta_{t}}\right)^{-1} .
\end{gathered}
$$

On the other hand,

$$
\begin{gathered}
f\left(a b^{-1}\right) \cdot f\left(c d^{-1}\right)=p_{\alpha_{1}} \ldots p_{\alpha_{n}}\left(p_{\beta_{m}}^{-1} \ldots p_{\beta_{1}}^{-1} \cdot p_{\beta_{1}} \ldots p_{\beta_{m}}\right) p_{\gamma_{m+1}} \ldots p_{\gamma_{k}}\left(p_{\delta_{1}} \ldots p_{\delta_{t}}\right)^{-1}= \\
=p_{\alpha_{1}} \ldots p_{\alpha_{n}} p_{\gamma_{m+1}} \ldots p_{\gamma_{k}}\left(p_{\delta_{1}} \ldots p_{\delta_{t}}\right)^{-1}=f\left(a b^{-1} \cdot c d^{-1}\right) .
\end{gathered}
$$

Case 2 is similar to case 1 . Consider case 3 . In this case there exists a positive integer $i$ such that $u_{\beta_{j}}=u_{\gamma_{j}}$ for every $j<i$ and $u_{\beta_{i}} \neq u_{\gamma_{i}}$. Observe that $f\left(a b^{-1} \cdot c d^{-1}\right)=f(0)=0$ and

$$
\begin{gathered}
f\left(a b^{-1}\right) \cdot f\left(c d^{-1}\right)=p_{\alpha_{1}} \ldots p_{\alpha_{n}} p_{\beta_{m}}^{-1} \ldots p_{\beta_{i}}^{-1}\left(p_{\beta_{i-1}}^{-1} \ldots p_{\beta_{1}}^{-1} \cdot p_{\beta_{1}} \ldots p_{\beta_{i-1}}\right) p_{\gamma_{i}} \ldots p_{\gamma_{k}}\left(p_{\delta_{1}} \ldots p_{\delta_{t}}\right)^{-1}= \\
=p_{\alpha_{1}} \ldots p_{\alpha_{n}} p_{\beta_{m}}^{-1} \ldots\left(p_{\beta_{i}}^{-1} \cdot p_{\gamma_{i}}\right) \ldots p_{\gamma_{k}}\left(p_{\delta_{1}} \ldots p_{\delta_{t}}\right)^{-1}=0=f\left(a b^{-1} \cdot c d^{-1}\right) .
\end{gathered}
$$

Hence $f$ is an isomorphism between semigroups $\left\langle C_{e}\right\rangle$ and $\mathcal{P}_{\left|C_{e}^{1} \backslash\{e\}\right|}$.

The following theorem describes the structure of a subsemigroup $D_{e}^{0}$ of an arbitrary GIS. 
Theorem 2. Let $E$ be any graph and $e \in E^{0}$. Then the semigroup $D_{e}^{0}$ is isomorphic to the Brandt $Q_{e}^{0}$-extension of the polycyclic monoid $\mathcal{P}_{\left|C_{e}^{1} \backslash\{e\}\right|}$.

Proof. Recall that $D_{e}=\left\{u v^{-1} \mid r(u)=r(v)=e\right\}$. The proof is based on the following obvious fact: each element $u \in I_{e}$ can be uniquely represented as follows: $u=u_{1} u_{2}$ where $u_{1} \in Q_{e}$ and $u_{2} \in C_{e}$ (here both $u_{1}$ and $u_{2}$ can be equal to $e$ ). By Theorem 1, the semigroup $\left\langle C_{e}\right\rangle$ is isomorphic to the polycyclic monoid $\mathcal{P}_{\left|C_{e}^{1} \backslash\{e\}\right|}$. Define the map $h: D_{e}^{0} \rightarrow B_{Q_{e}}^{0}\left(\mathcal{P}_{\left|C_{e}^{1} \backslash\{e\}\right|}\right)$ as follows: $h(0)=0$ and $h\left(u v^{-1}\right)=\left(u_{1}, f\left(u_{2} v_{2}^{-1}\right), v_{1}\right)$ for each non-zero element $u v^{-1}=$ $u_{1} u_{2}\left(v_{1} v_{2}\right)^{-1} \in D_{e}$ where $u_{1}, v_{1} \in Q_{e}, u_{2}, v_{2} \in C_{e}$ and $f$ is an isomorphism between semigroups $\left\langle C_{e}\right\rangle$ and $\mathcal{P}_{\left|C_{e}^{1} \backslash\{e\}\right|}$ defined in Theorem 1 . We remark that $f(e)=(e, 1, e)$. Suppose that $u \neq v$ for some paths $u, v \in I_{e}$. Then $u_{1} \neq v_{1}$ or $u_{2} \neq v_{2}$ which implies that the map $h$ is injective. Since for each non-zero element $\left(a, u v^{-1}, b\right) \in B_{Q_{e}}^{0}\left(\mathcal{P}_{\left|C_{e}^{1} \backslash\{e\}\right|}\right)$ we have

$$
h\left(a f^{-1}(u)\left(b f^{-1}(v)\right)^{-1}\right)=\left(a, f\left(f^{-1}(u) f^{-1}(v)^{-1}\right), b\right)=\left(a, f f^{-1}\left(u v^{-1}\right), b\right)=\left(a, u v^{-1}, b\right)
$$

the map $h$ is bijective. Now it remains to show that $h$ is a homomorphism. Fix any elements $a b^{-1}, c d^{-1} \in D_{e}$. Following the main idea of the proof we can uniquely represent elements $a, b, c, d \in I_{e}$ as follows: $a=a_{1} a_{2}, b=b_{1} b_{2}, c=c_{1} c_{2}$ and $d=d_{1} d_{2}$ where $a_{1}, b_{1}, c_{1}, d_{1} \in Q_{e}$ and $a_{2}, b_{2}, c_{2}, d_{2} \in C_{e}$. There are three cases to consider:

(1) There exists $w \in \operatorname{Path}(E)$ such that $a b^{-1} \cdot c d^{-1}=a w d^{-1}$, i.e., $c=b w$;

(2) there exists $w \in \operatorname{Path}(E)$ such that $a b^{-1} \cdot c d^{-1}=a(d w)^{-1}$, i.e., $b=c w$;

(3) $a b^{-1} \cdot c d^{-1}=0$.

Consider case 1 . Observe that $s(w)=r(b)=r(c)=r(w)$ which implies that $c_{1}=b_{1}$ and $c_{2}=b_{2} w$. Hence

$$
\begin{gathered}
h\left(a b^{-1}\right) \cdot h\left(c d^{-1}\right)=\left(a_{1}, f\left(a_{2} b_{2}^{-1}\right), b_{1}\right) \cdot\left(b_{1}, f\left(b_{2} w d_{2}^{-1}\right), d_{1}\right)=\left(a_{1}, f\left(a_{2} b_{2}^{-1}\right) \cdot f\left(b_{2} w d_{2}^{-1}\right), d_{1}\right)= \\
=\left(a_{1}, f\left(a_{2} b_{2}^{-1} \cdot b_{2} w d_{2}^{-1}\right), d_{1}\right)=\left(a_{1}, f\left(a_{2} w d_{2}^{-1}\right), d_{1}\right)=h\left(a w d^{-1}\right) .
\end{gathered}
$$

Consider case 2. Observe that $s(w)=r(c)=r(b)=r(w)$ which implies that $c_{1}=b_{1}$ and $b_{2}=c_{2} w$. Similar calculations as in case 1 show that $h\left(a b^{-1}\right) \cdot h\left(c d^{-1}\right)=h\left(a(d w)^{-1}\right)$.

Consider case 3. Observe that for each path $w \in \operatorname{Path}(E)$ neither $b=c w$ nor $c=b w$. Then one of the following two subcases holds:

(3.1) $b_{1} \neq c_{1}$;

(3.2) $b_{1}=c_{1}$, but for any $w \in \operatorname{Path}(E)$ neither $b_{2}=c_{2} w$ nor $c_{2}=b_{2} w$.

Consider subcase 3.1. Then

$$
h\left(a b^{-1}\right) \cdot h\left(c d^{-1}\right)=\left(a_{1}, f\left(a_{2} b_{2}^{-1}\right), b_{1}\right) \cdot\left(c_{1}, f\left(c_{2} d_{2}^{-1}\right), d_{1}\right)=0=f(0) .
$$

Consider subcase 3.2. Then

$$
\begin{aligned}
& h\left(a b^{-1}\right) \cdot h\left(c d^{-1}\right)=\left(a_{1}, f\left(a_{2} b_{2}^{-1}\right), b_{1}\right) \cdot\left(b_{1}, f\left(c_{2} d_{2}^{-1}\right), d_{1}\right)=\left(a_{1}, f\left(a_{2} b_{2}^{-1}\right) \cdot f\left(c_{2} d_{2}^{-1}\right), d_{1}\right)= \\
& =\left(a_{1}, f\left(a_{2} b_{2}^{-1} \cdot c_{2} d_{2}^{-1}\right), d_{1}\right)=\left(a_{1}, 0, d_{1}\right)=0=h(0) .
\end{aligned}
$$

Hence the map $h$ is an isomorphism between semigroups $D_{e}^{0}$ and $B_{Q_{e}}^{0}\left(\mathcal{P}_{\left|C_{e}^{1} \backslash\{e\}\right|}\right)$.

Graph $E$ is called acyclic at a vertex $e \in E^{0}$ if $C_{e}=\{e\}$. 
Corollary 2. Let $E$ be a graph which is acyclic at a vertex $e$. Then the subsemigroup $D_{e}^{0}$ of $G(E)$ is isomorphic to the semigroup of $I_{e} \times I_{e}$-matrix units $\mathcal{B}_{I_{e}}^{0}$.

Proof. Recall that by $B_{X}^{0}$ we denote the semigroup $B_{X}^{0}\left(\mathcal{P}_{0}\right)$. Since graph $E$ is acyclic at a vertex $e$ we obtain that $I_{e}=Q_{e}$ and $C_{e}=\{e\}$. By Theorem 2, the semigroup $D_{e}^{0}$ is isomorphic to the semigroup $B_{I_{e}}^{0}\left(\mathcal{P}_{0}\right)$.

By [22, Corollary 2], two non-zero elements $a b^{-1}$ and $c d^{-1}$ of a GIS $G(E)$ are $\mathcal{J}$-equivalent iff there exist elements $u, v \in \operatorname{Path}(E)$ such that $s(u)=r(a)=r(v)$ and $r(u)=r(c)=s(v)$. There exists a one to one correspondence between the set of strongly connected components of a graph $E$ and non-zero $\mathcal{J}$-classes of a GIS $G(E)$. More precisely, $J \cap E^{0}$ is a strongly connected component of a graph $E$ for each non-zero $\mathcal{J}$-class $J$ of $G(E)$. Therefore, by $J_{A}$ we denote a $\mathcal{J}$-class which contains a strongly connected component $A \subset E^{0}$.

Observe that for each strongly connected component $A$ of a graph $E, \mathcal{J}_{A}=\bigcup_{e \in A} D_{e}$. Hence Lemma 1 provides the following:

Corollary 3. For each strongly connected component $A$ of a graph $E$ the set $J_{A}^{0}=J_{A} \cup\{0\}$ is an inverse subsemigroup of $G(E)$.

Let $E$ be a graph and $X$ be any non-empty subset of $E^{0}$. By $E_{X}$ we denote the induced (by the set $X$ ) subgraph of the graph $E$, i.e., $E_{X}^{0}=X, E_{X}^{1}=\left\{x \in E^{1} \mid s(x) \in X\right.$ and $\left.r(x) \in X\right\}$ and source (resp., range) function $s_{X}$ (resp., $r_{X}$ ) of the graph $E_{X}$ is the restriction of the source function $s$ (resp., range function $r$ ) of the graph $E$ on the set $E_{X}^{1}$. Let $A$ be a strongly connected component of a graph E. Put

$$
\begin{gathered}
I_{A}=\{u \in \operatorname{Path}(E) \mid r(u) \in A\} ; \\
Q_{A}=\left\{u \in I_{A} \mid r(v) \notin A, \text { for each non-trivial prefix } v \text { of } u\right\} .
\end{gathered}
$$

Lemma 2. Let $A$ be a strongly connected component of a graph $E$ and $w$ be a path such that $s(w) \in A$ and $r(w) \in A$. Then $w \in \operatorname{Path}\left(E_{A}\right)$ where $E_{A}$ is an induced subgraph of $E$.

Proof. The proof is obvious if $w=e \in A$. Let $w=a_{1} \ldots a_{n}$ be a path of non-zero length such that $s(w)=s\left(a_{1}\right)=e_{1} \in A$ and $r(w)=r\left(a_{n}\right)=f \in A$. Put $s\left(a_{i}\right)=e_{i}$, for each $i \leq n$. Since vertices $e_{1}$ and $f$ belong to $A$ there exists a path $u$ such that $s(u)=f$ and $r(u)=e_{1}$. We claim that for each $i \leq n$ vertices $e_{i}$ belong to $A$. Indeed, put $x=a_{1} \ldots a_{i-1}$ and $y=a_{i} \ldots a_{n} u$. Then $s(x)=e_{1}, r(x)=e_{i}$ and $s(y)=e_{i}, r(y)=e_{1}$ which provides that $\left\{e_{i}\right\}_{i \leq n} \subseteq A$. Hence $a_{i} \in E_{A}^{1}$ for every $i \leq n$ and, as a consequence, $w \in \operatorname{Path}\left(E_{A}\right)$.

The following theorem describes the structure of a subsemigroup $J_{A}^{0}$ of $G(E)$ where $A$ is any strongly connected component of a graph $E$.

Theorem 3. Let $E$ be any graph and $A \subseteq E^{0}$ be a strongly connected component. Then the semigroup $J_{A}^{0}$ is isomorphic to a subsemigroup of the Brandt $Q_{A}^{0}$-extension of the graph inverse semigroup $G\left(E_{A}\right)$ over the induced subgraph $E_{A}$.

Proof. The proof of this theorem is based on the following fact which follows from Lemma 2. Each element $u \in I_{A}$ can be uniquely represented as follows: $u=u_{1} u_{2}$ where $u_{1} \in Q_{A}$ and $u_{2} \in \operatorname{Path}\left(E_{A}\right) \subset \operatorname{Path}(E)$. Observe that $u_{1}$ and $u_{2}$ could be equal to some vertex $e \in A$. Define the map $f: J_{A}^{0} \rightarrow B_{Q_{A}}^{0}\left(G\left(E_{A}\right)\right)$ by the following way: $f(0)=0$ and for each non-zero element $u v^{-1}=u_{1} u_{2}\left(v_{1} v_{2}\right)^{-1} \in G(E)$ where $u_{1}, v_{1} \in Q_{A}$ and $u_{2}, v_{2} \in \operatorname{Path}\left(E_{A}\right) \subset \operatorname{Path}(E)$ 
put $f\left(u v^{-1}\right)=\left(u_{1}, u_{2} v_{2}^{-1}, v_{1}\right)$. The injectivity of the map $f$ is straightforward. Next we show that the map $f$ is a homomorphism. Fix any elements $a b^{-1}, c d^{-1} \in J_{A}$. Following the main idea of the proof we can uniquely represent elements $a, b, c, d \in I_{A}$ as follows: $a=a_{1} a_{2}, b=b_{1} b_{2}, c=c_{1} c_{2}$ and $d=d_{1} d_{2}$ where $a_{1}, b_{1}, c_{1}, d_{1} \in Q_{A}$ and $a_{2}, b_{2}, c_{2}, d_{2} \in G\left(E_{A}\right)$. There are three cases to consider:

(1) there exists $w \in \operatorname{Path}(E) \operatorname{such}$ that $a b^{-1} \cdot c d^{-1}=a w d^{-1}$, i.e., $c=b w$;

(2) there exists $w \in \operatorname{Path}(E)$ such that $a b^{-1} \cdot c d^{-1}=a(d w)^{-1}$, i.e., $b=c w$;

(3) $a b^{-1} \cdot c d^{-1}=0$.

Consider case 1. Observe that $s(w)=r(b) \in A$ and $r(w)=r(c) \in A$. By Lemma 2, $w \in \operatorname{Path}\left(E_{A}\right)$ which implies that $c_{1}=b_{1}$ and $c_{2}=b_{2} w$. Hence

$$
\begin{gathered}
f\left(a b^{-1}\right) \cdot f\left(c d^{-1}\right)=\left(a_{1}, a_{2} b_{2}^{-1}, b_{1}\right) \cdot\left(b_{1}, b_{2} w d_{2}^{-1}, d_{1}\right)=\left(a_{1}, a_{2} b_{2}^{-1} \cdot b_{2} w d_{2}^{-1}, d_{1}\right)= \\
=\left(a_{1}, a_{2} w d_{2}^{-1}, d_{1}\right)=f\left(a w d^{-1}\right) .
\end{gathered}
$$

Consider case 2. Observe that $s(w)=r(c) \in A$ and $r(w)=r(b) \in A$. By Lemma 2, $w \in \operatorname{Path}\left(E_{A}\right)$ which implies that $c_{1}=b_{1}$ and $b_{2}=c_{2} w$. Similar calculations as in case 1 show that $f\left(a b^{-1}\right) \cdot f\left(c d^{-1}\right)=f\left(a(d w)^{-1}\right)$.

Consider case 3. Observe that neither $b=c w$ nor $c=b w$. Then one of the following two subcases holds:

(3.1) $b_{1} \neq c_{1}$;

(3.2) $b_{1}=c_{1}$, but for any $w \in \operatorname{Path}(E)$ neither $b_{2}=c_{2} w$ nor $c_{2}=b_{2} w$.

Consider subcase 3.1. Then $f\left(a b^{-1}\right) \cdot f\left(c d^{-1}\right)=\left(a_{1}, a_{2} b_{2}^{-1}, b_{1}\right) \cdot\left(c_{1}, c_{2} d_{2}^{-1}, d_{1}\right)=0=f(0)$. Consider subcase 3.2. Then

$$
\begin{aligned}
& f\left(a b^{-1}\right) \cdot f\left(c d^{-1}\right)=\left(a_{1}, a_{2} b_{2}^{-1}, b_{1}\right) \cdot\left(b_{1}, c_{2} d_{2}^{-1}, d_{1}\right)= \\
& =\left(a_{1}, a_{2} b_{2}^{-1} \cdot c_{2} d_{2}^{-1}, d_{1}\right)=\left(a_{1}, 0, d_{1}\right)=0=f(0) .
\end{aligned}
$$

Hence the map $f$ is an isomorphic embedding of the semigroup $J_{A}^{0}$ into $B_{Q_{A}}^{0}\left(G\left(E_{A}\right)\right)$.

3. A global structure of graph inverse semigroups. By $\mathcal{A}$ we denote the set of all strongly connected components of a graph $E$. The set $\mathcal{A}$ admits a natural partial order $\leq$ : for each $X, Y \in \mathcal{A}, X \leq Y$ iff there exists a path $u \in \operatorname{Path}(E)$ such that $s(u) \in Y$ and $r(u) \in X$.

Theorem 4. For any graph $E$ the following statements hold:

(1) $G(E)=\bigcup_{X \in \mathcal{A}} J_{X}^{0}$;

(2) $J_{X}^{0}$ is isomorphic to a subsemigroup of $B_{Q_{X}}^{0}\left(G\left(E_{X}\right)\right)$, for each $X \in \mathcal{A}$.

(3) $J_{X}^{0} \cap J_{Y}^{0}=\{0\}$ for each distinct elements $X, Y \in \mathcal{A}$;

(4) if $X \leq Y$ then $J_{X}^{0} \cdot J_{Y}^{0} \cup J_{Y}^{0} \cdot J_{X}^{0} \subseteq J_{X}^{0}$;

(5) if $X \not \leq Y$ and $Y \not X X$ then $J_{X}^{0} \cdot J_{Y}^{0} \cup J_{Y}^{0} \cdot J_{X}^{0} \subseteq\{0\}$.

Proof. Statements 1 and 3 follows from the fact that $\mathcal{J}$ is an equivalence relation. Statement 2 follows from Theorem 3.

Consider statement 4. Assume that $X, Y \in \mathcal{A}$ and $X \leq Y$. Fix any elements $a b^{-1} \in J_{X}^{0}$ and $c d^{-1} \in J_{Y}^{0}$. Observe that the case $a b^{-1} \cdot c d^{-1}=0$ is trivial, because $0 \in J_{X}^{0}$. Suppose that 
$a b^{-1} \cdot c d^{-1} \neq 0$. In this case there exists a path $w$ such that either $c=b w$ or $b=c w$. If $b=c w$ then $a b^{-1} \cdot c d^{-1}=a(d w)^{-1}$ and $r(d w)=r(w)=r(b)=r(a) \in X$. Hence $a(d w)^{-1} \in J_{X}$. If $c=b w$ then $s(w)=r(b) \in X$ and $r(w)=r(c) \in Y$ which implies that $Y \leq X$. Since the order $\leq$ is antisymmetric we obtain that $X=Y$. Hence Corollary 3 provides that $a b^{-1} \cdot c d^{-1} \in J_{X}$.

Consider statement 5 . Assume that $X \not \leq Y$ and $Y \not X X$. Fix any elements $a b^{-1} \in J_{X}^{0}$ and $c d^{-1} \in J_{Y}^{0}$. We claim that neither $b$ is a prefix of $c$ nor $c$ is a prefix of $b$. Indeed, if $b$ is a prefix of $c$, i.e., $c=b w$ for some path $w$. Then $s(w)=r(b) \in X$ and $r(w)=r(c) \in Y$ witnessing that $Y \leq X$ which contradicts to the assumption. If $c$ is a prefix of $b$, i.e., $b=c w$ for some path $w$. Then $s(w)=r(c) \in Y$ and $r(w)=r(b) \in X$ witnessing that $X \leq Y$ which contradicts to the assumption. Hence $a b^{-1} \cdot c d^{-1}=0$.

The proof of the following lemma follows from the definition of Green's relations $\mathcal{D}$ and $\mathcal{J}$.

Lemma 3. For a graph inverse semigroup $G(E)$ the following conditions are equivalent:

(1) relations $\mathcal{J}$ and $\mathcal{D}$ coincide on $G(E)$;

(2) graph $E$ is acyclic.

Now we apply our results to graph inverse semigroups over acyclic graphs. Observe that each strongly connected component of an acyclic graph $E$ coincides with some vertex $e \in E^{0}$. Hence each acyclic graph $E$ admits a natural partial order $\leq$ on the set $E^{0}$. For each $e, f \in E^{0}$, $e \leq f$ iff there exists a path $u$ such that $s(u)=f$ and $r(u)=e$. The following theorem describes the structure of graph inverse semigroups over acyclic graphs.

Theorem 5. Let $E$ be an acyclic graph. Then the following statements hold:

(1) $G(E)=\bigcup_{e \in E^{0}} D_{e}^{0}$;

(2) $D_{e}^{0}$ is isomorphic to the semigroup of $I_{e} \times I_{e}$-matrix units $\mathcal{B}_{I_{e}}^{0}$, for each vertex $e \in E^{0}$;

(3) $D_{e}^{0} \cap D_{f}^{0}=\{0\}$, for each distinct vertices $e, f \in E^{0}$;

(4) If $e \leq f$ then $D_{e}^{0} \cdot D_{f}^{0} \cup D_{f}^{0} \cdot D_{e}^{0} \subseteq D_{e}^{0}$;

(5) If $e \not \leq f$ and $f \not \leq e$ then $D_{e}^{0} \cdot D_{f}^{0} \cup D_{f}^{0} \cdot D_{e}^{0}=\{0\}$.

Proof. Statements 1 and 3 are obvious. Statement 2 follows from Corollary 2. Statement 4 (resp., 5) follows from Lemma 3 and statement 4 (resp., 5) of Theorem 4.

Acknowledgements. The author acknowledges the referee for his comments and suggestions.

\section{REFERENCES}

1. G. Abrams and G. Aranda Pino, The Leavitt path algebra of a graph, J. Algebra 293, (2005), 319-334.

2. Amal Alali, N.D. Gilbert, Closed inverse subsemigroups of graph inverse semigroups, Communications in Algebra, 45 (11), (2017), 4667-4678.

3. P. Ara, M. Moreno, E. Pardo, Non-stable K-theory for graph algebras, Algebr. Represent. Th., 10 (2007), 157-178. 
4. S. Bardyla, Classifying locally compact semitopological polycyclic monoids, Math. Bulletin of the Shevchenko Scientific Society, 13 (2016), 21-28.

5. S. Bardyla, On universal objects in the class of graph inverse semigroups, European Journal of Mathematics, in press, DOI: 10.1007/s40879-018-0300-7.

6. S. Bardyla, On locally compact topological graph inverse semigroups, preprint, (2017), arXiv:1706.08594.

7. S. Bardyla, Embeddings of graph inverse semigroups into compact-like topological semigroups, preprint, (2019), arXiv:1810.09169.

8. S. Bardyla, On locally compact semitopological graph inverse semigroups, Mat. Stud., 49 (2018), №1, 19-28.

9. S. Bardyla, O. Gutik, On a semitopological polycyclic monoid, Algebra Discr. Math., 21 (2016), №2, $163-183$.

10. S. Bardyla, O. Gutik, On a complete topological inverse polycyclic monoid, Carpathian Math. Publ., 8 (2016), №2, 183-194.

11. A.H. Clifford, G.B. Preston, The Algebraic Theory of Semigroups, Vol. I and II, Amer. Math. Soc. Surveys, 7, Providence, R.I., 1961 and 1967.

12. J. Cuntz, W. Krieger, A class of $C^{*}$-algebras and topological Markov chains, Invent. Math., 56 (1980), 251-268.

13. O. Gutik, On the dichotomy of the locally compact semitopological bicyclic monoid with adjoined zero, Visn. L'viv. Univ., Ser. Mekh.-Mat., 80 (2015), 33-41.

14. O. Gutik, K. Pavlyk, On Brandt $\lambda^{0}$-extensions of semigroups with zero, Mat. Metody Fiz.-Mech. Polya, 49 (2006), №3, 26-40.

15. O. Gutik, D. Repovs, On the Brandt $\lambda^{0}$-extensions of monoids with zero, Semigroup Forum, 80, (2010), $8-32$.

16. D.G. Jones, Polycyclic monoids and their generalizations, PhD Thesis, Heriot-Watt University, 2011.

17. D.G. Jones, M.V. Lawson, Graph inverse semigroups: Their characterization and completion, J. Algebra, 409 (2014), 444-473.

18. A. Kumjian, D. Pask, I. Raeburn, Cuntz-Krieger algebras of directed graphs, Pacific J. Math., 184 (1998), $161-174$.

19. M. Lawson, Inverse Semigroups. The Theory of Partial Symmetries, Singapore: World Scientific, 1998.

20. M.V. Lawson, Primitive partial permutation representations of the polycyclic monoids and branching function systems, Period. Math. Hungar., 58 (2009), 189-207.

21. J. Meakin, M. Sapir, Congruences on free monoids and submonoids of polycyclic monoids, J. Austral. Math. Soc. Ser. A, 54 (2009), 236-253.

22. Z. Mesyan, J.D. Mitchell, The structure of a graph inverse semigroup, Semigroup Forum, 93 (2016), $111-130$.

23. Z. Mesyan, J.D. Mitchell, M. Morayne, Y.H. Péresse, Topological graph inverse semigroups, Topology and its Applications, 208 (2016), 106-126.

24. M. Nivat, J.-F. Perrot, Une généralisation du monoide bicyclique, C. R. Acad. Sci., Paris, Sér. A, 271 (1970), 824-827.

25. A. Paterson, Graph inverse semigroups, groupoids and their $C^{*}$-algebras, J. Operator Theory, 48 (2002), №3, suppl., 645-662.

Institute of Mathematics, Kurt Gödel Research Center, Vienna, Austria sbardyla@yahoo.com 